\title{
Hepatitis B notifications in a vaccinated cohort of Aboriginal people in the Kimberley region
}

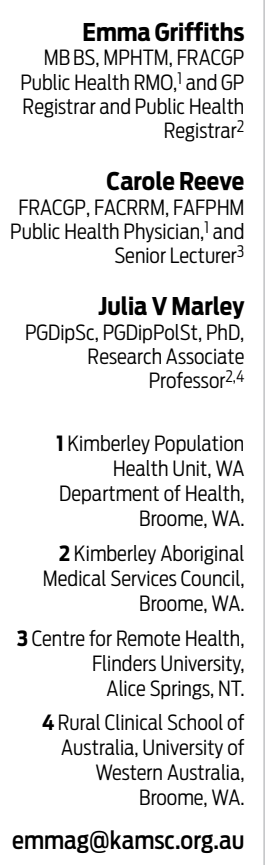

MJA 2014; 201: 343-346 doi: 10.5694/mjal3.00255
. epatitis B virus (HBV) infection constitutes a considerable international health problem. ${ }^{1}$ In Australia, an estimated 218000 people are living with chronic HBV infection. ${ }^{2}$ Aboriginal and Torres Strait Islander people comprise about $2.5 \%$ of the Australian population but account for an estimated $9.3 \%$ of cases of chronic HBV infection. ${ }^{2}$

The probability of chronic HBV infection is inversely proportional to the age at acquisition (up to $90 \%$ in infancy), with infants of mothers with chronic infection at risk of vertical transmission. ${ }^{1}$ Longitudinal studies have demonstrated that the introduction of the HBV vaccine has dramatically reduced HBV prevalence in endemic areas. ${ }^{3-5}$ Northern Territory data suggest a similar reduction in Australia. ${ }^{6}$

However, infections after full vaccination (considered to be a vaccine failure) have been well documented, largely in high prevalence countries with increased statistical likelihood of breakthrough infections, and predominantly where the mother had known chronic infection with high infectivity. Most cases were thought to have occurred through vertical transmission at delivery. 4,5,7 In an Australian study of children born to mothers with chronic infection, perinatal transmission occurred in 3\% and was limited to children of mothers with high viral load or hepatitis $B$ e antigen positivity (a serological marker of high infectivity). ${ }^{8}$

The Kimberley is a region of north Western Australia with a relatively small population (29296 at the time of the 2006 census) and a high proportion of Aboriginal residents (42\%). ${ }^{9}$ The background prevalence of chronic HBV infection in 1986 was $8 \%, 10$ meeting the World Health Organization criteria for high endemnicity. ${ }^{1}$ Routine vaccination against HBV was introduced in the Kimberley in 1988. We would therefore expect most of the population

\section{Abstract}

Objectives: To identify cases of hepatitis B infection after vaccination in Kimberley residents and determine maternal serostatus as a potential indicator of mode of transmission.

Design and participants: Retrospective review of Kimberley residents with notified hepatitis $B$ infection using records of vaccination history and child and maternal serology.

Main outcome measures: Confirmed cases of hepatitis B infection after vaccination; chronic infection in cases of hepatitis $B$ infection after vaccination; maternal serostatus in confirmed cases of hepatitis B infection after vaccination.

Results: From 1 January 1984 to 31 March 2011, we identified 17 cases of Aboriginal residents with hepatitis $B$ infection after vaccination (10 chronic infections, five not defined and two cleared). In six patients, maternal chronic infection had been identified at some stage, raising the possibility of vertical transmission. In seven patients, maternal serology or evidence of subsequent acquisition suggested that horizontal transmission and therefore vaccination failure was likely. For four patients, there was inadequate information to assess possible mode of transmission.

Conclusions: Hepatitis B infection after full vaccination is not limited to children of mothers with active infection. Further undiagnosed infections in the Kimberley are likely, and active monitoring to detect the extent and likely cause of hepatitis B infection in vaccinated children is needed. In children where vertical transmission is a risk, prospective follow-up is warranted.

born in the Kimberley on or after 1 January 1989 to be fully vaccinated and the epidemiological pattern to transition to that seen in lower endemnicity areas, with the majority of cases occurring in high-risk adults.

Concerns have been raised about the effectiveness of the HBV vaccine within Aboriginal and Torres Strait Islander communities. ${ }^{11-13}$ The WA Country Health Service (WACHS) Kimberley Population Health Unit was notified of several cases where HBV infection followed full vaccination. We aimed to document all such cases in the region from 1984 to 2011 and sought details on maternal sero$\log y$ for further information about likely modes of transmission.

\section{Methods}

\section{Case definition}

Cases were defined as Kimberley residents with a notification of HBV infection to the WA Notifiable Infectious Diseases Database from 1 January 1984 to 31 March 2011 and a history of full primary vaccination as per the then recommended schedule (at birth, 1 month and 3 months), ${ }^{14}$ including those fully vaccinated before the regional vaccination program. The search was restricted to residents born on or after 1970 who were targeted by the school program or vaccinated from birth. Children with delayed (first dose given after the first week of life) or incomplete (less than three doses) vaccination were excluded. Maternal serology was sought, and where maternal HBV chronic infection was identified, records of hepatitis B immunoglobulin (HBIG) administration were checked to determine if missed HBIG doses could have contributed to vaccine failure.

Where follow-up testing was available, patients were considered to have chronic HBV infection if HBV surface antigen (HBsAg) or HBV DNA remained detectable in the blood after 6 months or if HBsAg was detected in a patient shown to be immunoglobulin $\mathrm{M}$ anti-hepatitis B core antigen negative as per the Australian Government Department of Health criteria. ${ }^{15}$ 


\section{Determination of the final study cohort}

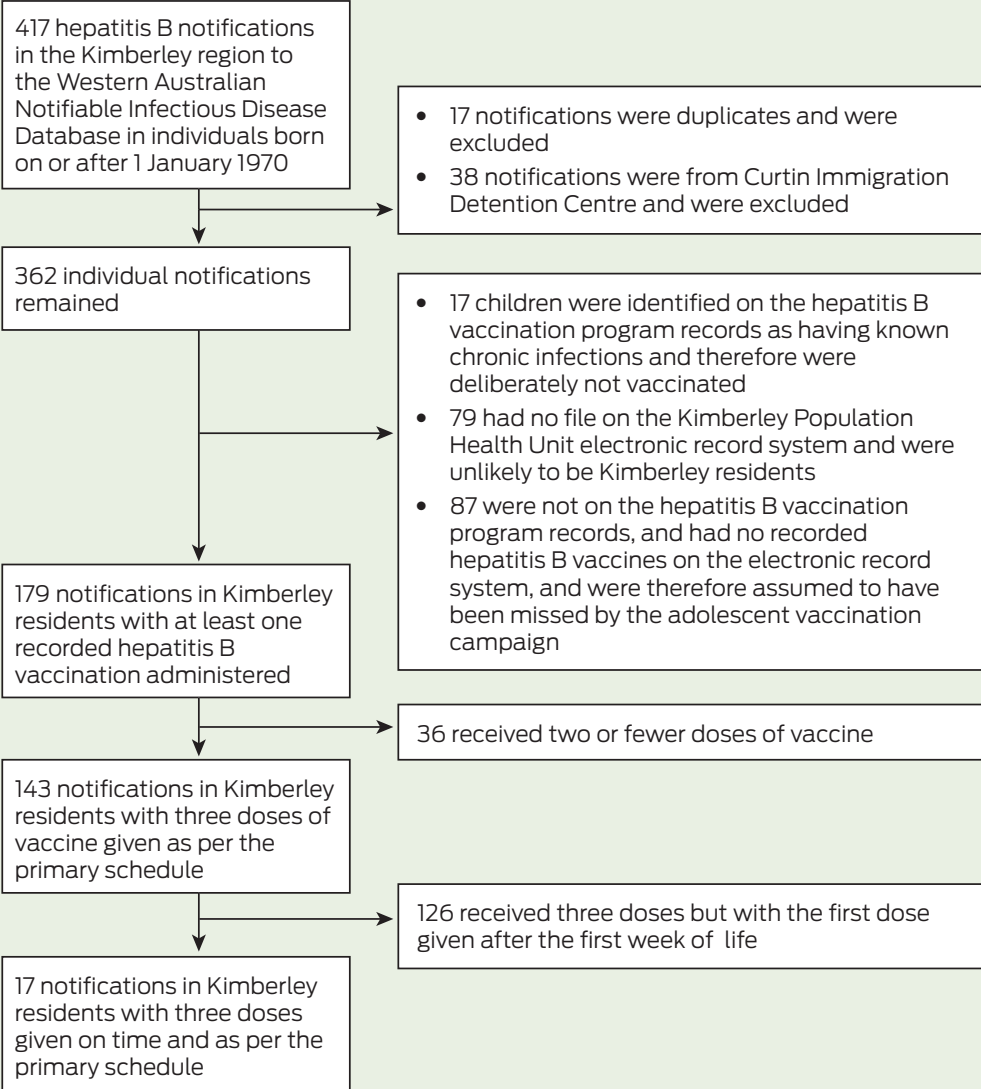

\section{Data extraction}

Information on notified cases of HBV infection from the Kimberley region was extracted from the WA Notifiable Infectious Diseases Database. Notifications from testing laboratories began in 2000 and have been mandatory since 2006. Before this, notification was practitioner dependent.

The final cohort was determined by cross-referencing this information with available immunisation history and hepatitis serology records (Box 1). Records of childhood vaccination were taken from the WA Department of Health country public hospital database (Health Care and Related Information System) and paper records held by the Kimberley Population Health Unit. Child and maternal hepatitis serology results were obtained from patient electronic records (Communicare [Communicare Systems] and MMex [University of WA]) and WA Country Health Service paper file reviews.

\section{Ethics approval}

Ethics approval was obtained from the WACHS Human Research Ethics Committee and the WA Aboriginal Health Information and Ethics Committee. The project was supported by the Kimberley Aboriginal Health Planning Forum Research Sub-Committee.

\section{Results}

After identifying 417 Kimberley residents with notifications of HBV infection, 400 were excluded for not meeting the case definition (Box 1). All 17 remaining patients were of Aboriginal descent. Of these, 10 patients had evidence of chronic infection, five had unknown status and two had cleared the virus (Box 2). In seven patients, vertical transmission was unlikely because maternal hepatitis serology results were negative after the delivery or indicated antenatal immunity (cases 9, 11, 14, 16 and 17), or there was evidence of subsequent acquisition (cases 12 and 8). In six patients, vertical transmission was possible because of known maternal chronic infection at delivery (cases 1 , 7,13 and 15) or after delivery (cases 6 and 10). For four individuals, there was insufficient information available to determine the risk of vertical transmission (cases 2, 3, 4 and 5).

In three of the four patients for whom maternal chronic infection had been identified (cases 1, 7 and 15), HBIG was documented as given according to protocol. Local notes did not document the administration of HBIG for the fourth patient (case 13); however, the child was born at the tertiary obstetric referral hospital and therefore would likely have received immunoglobulin.

\section{Discussion}

Our study described 17 cases of Aboriginal Kimberley residents with HBV infection after full vaccination and a high rate of chronic infection. For 13 patients, the mother was not known to have chronic HBV infection during pregnancy. In seven of these patients, correlation with maternal serology suggests that transmission in early childhood was likely. This emphasises the importance of prevention of transmission from household contacts through early diagnosis of positive cases and the need for advice on prevention (eg, avoiding shared use of razors and toothbrushes, and coverage of skin sores).

It is noteworthy that with the exception of two people diagnosed as part of contact tracing, all patients were diagnosed at 13 years of age or older (presumably with the commencement of sexual activity and screening for sexually transmissible infection). This reflects the previous lack of a formalised follow-up process for high-risk children, which may have allowed transmission to household members and early sexual partners.

Our study had some notable limitations. As HBV testing rates are unknown, it is impossible to estimate the rate of vaccine failure per population of vaccinated individuals, limiting comparisons to other populations. The nature of the notification system also means that individuals vaccinated in the Kimberley region who relocate and are subsequently 
diagnosed with HBV infection would not be identified. Some paper records were archived offsite or otherwise unavailable.

A large study from the NT has demonstrated a reduction over time in HBV prevalence in birthing mothers who should have received newborn vaccination according to their date of birth; however, the prevalence was higher in mothers from very remote (3.1\%) compared with remote $(1.3 \%)$ and outer regional $(0.6 \%)$ areas. ${ }^{6}$ This highlights the importance of monitoring vaccine uptake and effectiveness.

In Australia, concerns have been expressed regarding the relative effectiveness of the HBV vaccine in Aboriginal cohorts, particularly in remote areas. An NT study found four of 37 vaccinated adolescents had chronic HBV infection after full vaccination. ${ }^{13}$ In a North Queensland study of 239 fully vaccinated children, 15 had evidence of natural infection, four had chronic disease and 113 lacked evidence of serological immunity. ${ }^{12}$ Those lacking evidence of immunity were given a booster vaccine and followed up for seroconversion; of these, 18 (16\%) lacked an anamnestic response, which has been used as a surrogate measure of protection from vaccination.

Contributing factors to vaccine failure may include patient factors such as poor nutrition or medical comorbidity impacting on immune response to vaccination, or vaccine factors such as cold chain breaches. Significant cold chain breaches have been demonstrated in remote Australian areas, ${ }^{16}$ although the extent of this in the Kimberley over the study period is not known. Genetic factors may also be relevant, as genotyping of HBV samples from the NT has demonstrated an unusual HBV subgenotype found only in Aboriginal and Torres Strait Islander people and it has been postulated that viral serotype may influence response to vaccination. ${ }^{17,18}$

Our results demonstrate the need for ongoing surveillance of HBV vaccine effectiveness in this population, follow-up of high-risk children and best-practice antenatal care. This is a relatively small case series from a small population, but is likely an underestimation, as not all vaccinated

2 Hepatitis B notifications in Kimberley region residents fully vaccinated from birth, 1984-2011

\begin{tabular}{|c|c|c|c|c|}
\hline Case & Maternal serostatus* & $\begin{array}{l}\text { HBIG given if mother known } \\
\text { to have chronic infection }\end{array}$ & $\begin{array}{c}\text { Age at } \\
\text { notification, years }\end{array}$ & $\begin{array}{l}\text { Status at follow- } \\
\text { up testing }\end{array}$ \\
\hline 1 & Positive antenatally & Yes & $13-15$ & Chronic carrier \\
\hline 2 & Unknown & & $\geqslant 19$ & Unknown \\
\hline 3 & Unknown & & $13-15$ & Unknown \\
\hline 4 & Immune postnatally & & $\geqslant 19$ & Chronic carrier \\
\hline 5 & Unknown & & $16-18$ & Unknown \\
\hline 6 & Positive postnatally & & $13-15$ & Unknown \\
\hline 7 & Positive antenatally & Yes & $16-18$ & Chronic carrier \\
\hline 8 & Negative postnatally & & $16-18$ & Cleared $^{\dagger}$ \\
\hline 9 & Immune antenatally & & $\geqslant 19$ & Chronic carrier \\
\hline 10 & Positive postnatally & & $\geqslant 19$ & Chronic carrier \\
\hline 11 & Negative postnatally & & $16-18$ & Chronic carrier \\
\hline 12 & Unknown & & $13-15$ & Cleared $¥$ \\
\hline 13 & Positive antenatally & Unknown & $16-18$ & Chronic carrier \\
\hline 14 & Negative postnatally & & $13-15$ & Chronic carrier \\
\hline 15 & Positive antenatally & Yes & $13-15$ & Chronic carrier \\
\hline 16 & Negative postnatally & & $\leqslant 12$ & Unknown \\
\hline 17 & Negative postnatally & & $\leqslant 12$ & Chronic carrier \\
\hline
\end{tabular}

$\mathrm{HBIG}=$ hepatitis B immunoglobulin, given at delivery in high-risk cases. * Positive = hepatitis B surface antigen positive; immune = hepatitis B surface antigen negative and hepatitis B surface antibody positive; negative = hepatitis B surface antigen and hepatitis B surface antibody negative. † Case 8 known to be negative in 1992, indicating subsequent acquisition (after 3 years of age). $\ddagger$ Case 12 known to be immunoglobulin $M$ hepatitis $B$ core antibody positive in 2006 , indicating acquisition at this time. \$Case 13 born at tertiary obstetric referral hospital and likely to have been given HBIG.

individuals will have had subsequent HBV testing and some patients may have been tested and notified outside of the Kimberley region.

Following this audit, existing local protocols and strategies have been reviewed and additional local guidelines developed in collaboration between regional Aboriginal community controlled services and state government health services. ${ }^{19}$ There are ongoing and future plans for retrospective audit and followup of high-risk children, prospective correlation of all new notifications of hepatitis $B$ with vaccination history, and audit of practitioner testing behaviour to evaluate the implementation of the new regional HBV protocols.

Acknowledgements: We thank David Atkinson and Philippa Chidgzey for their critical review of this article. We thank the WACHS for providing the data used in this study.

Competing interests: No relevant disclosures.

Received 21 Dec 2013, accepted 20 May 2014.

1 Shepard CW, Simard EP, Finelli L, et al. Hepatitis $B$ virus infection: epidemiology and vaccination. Epidemiol Rev 2006; 28: 112-125.

2 MacLachlan JH, Allard N, Towell V, Cowie BC. The burden of chronic hepatitis B virus infection in Australia, 2011. Aust N Z J Public Health 2013; 37: 416-422.

$3 \mathrm{Ni}$ YH, Chang MH, Huang LM, et al. Hepatitis $B$ virus infection in children and adolescents in a hyperendemic area: 15 years after mass hepatitis B vaccination. Ann Intern Med 2001; 135: 796-800.

4 Poovorawan $Y$, Chongsrisawat $\mathrm{V}$ Theamboonlers A, et al. Long-term benefit of hepatitis $B$ vaccination among children in Thailand with transient hepatitis B virus infection who were born to hepatitis B surface antigen-positive mothers. J Infect Dis 2009; 200: 33-38.

5 Mendy M, Peterson I, Hossin S, et al. Observational study of vaccine efficacy 24 years after the start of hepatitis $B$ vaccination in two Gambian villages: no need for a booster dose. PLOS One 2013; 8: e58029.

6 Liu B, Guthridge S, Li SQ, et al. The end of the Australia antigen? An ecological study of the impact of universal newborn hepatitis $B$ vaccination two decades on. Vaccine 2012; 30 : 7309-7314.

7 Wu TW, Lin HH, Wang LY. Chronic hepatitis B infection in adolescents who received primary infantile vaccination. Hepatology 2013; 57: 37-45.

8 Wiseman E, Fraser MA, Holden S, et al. Perinatal transmission of hepatitis B virus: an Australian experience. Med J Aust 2009; 190: 489-492.

9 Australian Bureau of Statistics. 2006 Census Community Profiles. Kimberley. Code 545 (SD). Basic community profile [spreadsheet]. Canberra: ABS, 2007. (ABS Cat. No. 2001.0.) http://www.censusdata.abs.gov.au/census services/getproduct/census/2006/commun ityprofile/545?opendocument \&navpos $=220$ (accessed May 2014). 
10 Holman CD, Quadros CF, Bucens MR, Reid PM. Occurrence and distribution of hepatitis $B$ infection in the aboriginal population of Western Australia. Aust N Z J Med 1987; 17 518-525.

11 Hanna JN, Sexton WL, Faoagali JL, et al. Immunity to hepatitis B, poliomyelitis and measles in fully vaccinated Aboriginal and Torres Strait Island children. J Paediatr Child Health 1995; 31: 345-349.

12 Hanna JN, Faoagali JL, Buda PJ, Sheridan JW Further observations on the immune response to recombinant hepatitis $B$ vaccine after administration to Aboriginal and Torres Strait Island children. J Paediatr Child Health 1997; 33: $67-70$.
13 Dent E, Selvey CE, Bell A, et al. Incomplete protection against hepatitis $B$ among remote Aboriginal adolescents despite full vaccination in infancy. Commun Dis Intell Q Rep 2010; 34: 435-439.

14 Roberts DW; Health Department of Western Australia. Hepatitis B baby immunisation programme [circular]. 17 Dec 1986. http://www. health.wa.gov.au/circularsnew/pdfs/5780.pdf (accessed Aug 2013).

15 Department of Health and Ageing, Australian Government. Public Health Laboratory Network. Hepatitis B laboratory case definition (LCD). 2010. http://www.health.gov.au/ internet/main/publishing.nsf/Content/cdaphlncd-hepb (accessed Aug 2013)
16 Miller NC, Harris MF Are childhood immunization programmes in Australia at risk? Investigation of the cold chain in the Northern Territory. Bull World Health Organ 1994; 72: 401-408.

17 Davies J, Littlejohn M, Locarnini SA, et al. Molecular epidemiology of hepatitis $B$ in the Indigenous people of northern Australia. J Gastroenterol Hepatol 2013; 28: 1234-1241.

18 Littlejohn M. Davies J, Yuen L, et al. Molecular virology of hepatitis B virus, sub-genotype C4 in northern Australian Indigenous populations. J Med Virol 2014; 86: 695-706.

19 Kimberley Aboriginal Medical Services Council, WA Country Health Service Kimberley. Hepatitis B. 2012. http://resources.kamsc.org.au/ downloads/cd_hepb.pdf (accessed Dec 2013). 